\title{
Virtualización de la educación media superior y superior en México: cambios y realidades
}

\section{Virtualization of higher and higher middle education in Mexico: changes and realities}

\author{
GALLEGOS-GALINDO, Heriberto †*, AGUILERA-GUTIÉRREZ, Enrique, DOMÍNGUEZ-PIZANO, \\ Luis y CASTRO-VALENCIA, Alan
}

Universidad Politécnica de Juventino Rosas, México.

ID $1^{\mathrm{er}}$ Autor: Heriberto, Gallegos-Galindo / ORC ID: 0000-0002-5199-731X

ID $1^{\mathrm{er}}$ Coautor: Enrique, Aguilera-Gutiérrez / ORC ID: 0000-0001-7126-1898

ID $2^{\text {do }}$ Coautor: Luis, Domínguez-Pizano / ORC ID: 0000-0003-2175-5966

ID $3^{\text {er }}$ Coautor: Alan, Castro-Valencia / ORC ID: 0000-0002-5388-3148

DOI: $10.35429 /$ JOTE.2020.11.4.1.7

Recibido Enero 10, 2020; Aceptado Marzo 30, 2020

\section{Resumen}

La virtualización, que llegó a partir de la implementación de las computadoras, ha venido a transformar diferentes procesos en la vida diaria, incluyendo la educación. México es uno de los países que ha adoptado dentro de su sistema a la virtualización como una medida efectiva para lograr una mejor cobertura del estudiantado y disminuir la brecha educativa. Además de esto, se ha brindado acceso a Internet en diferentes puntos clave a fin de disminuir la brecha digital. Los cambios tecnológicos han traido consigo una gran mejora a los sistemas educativos, aunque algunos consideran que no son realmente efectivos. La mayoría de estos cambios se han dado en dos áreas principales de la educación: media superior y superior, es aquí donde actualmente se tiene mayor presencia y conforme pasan los años se tendrá una mayor presencia, ya que la virtualización nos permitirá mejorar las opciones que hay hoy en día. La presente investigación muestra una cronología de los cambios que ha traído la virtualización a la educación en México, así como la realidad y los cambios que esto ha generado.

Virtualización, Educación, México

\begin{abstract}
Virtualization, which came from the implementation of computers, has come to transform different processes in daily life, including education. Mexico is one of the countries that has adopted virtualization within its system as an effective measure to achieve better student coverage and reduce the educational gap. In addition to this, Internet access has been provided at different key points in order to reduce the digital divide. Technological changes have brought a great improvement to educational systems, although some consider that they are not really effective. Most of these changes have occurred in two main areas of education: upper and upper secondary, this is where we currently have the greatest presence and as the years go by we will have a greater presence, since virtualization will allow us to improve the options that there today. The present investigation shows a chronology of the changes that virtualization has brought to education in Mexico, as well as the reality and the changes that this has generated.
\end{abstract}

Virtualization, Education, Mexico

Citación: GALLEGOS-GALINDO, Heriberto, AGUILERA-GUTIÉRREZ, Enrique, DOMÍNGUEZ-PIZANO, Luis y CASTRO-VALENCIA, Alan. Virtualización de la educación media superior y superior en México: cambios y realidades. Revista de Educación Técnica. 2020. 4-11:1-7.

\footnotetext{
*Correspondencia al Autor (Correo Electrónico: hgalindo_ptc@upjr.edu.mx)

$\dagger$ Investigador contribuyendo como primer autor.
} 


\section{Introducción}

La palabra educación proviene del latín "educatio, -ōnis.", que significa "Instrucción por medio de la acción docente".

El sistema educativo en México ha atravesado por diferentes etapas, y cada una, que también pueden ser vistas como paradigmas, ha establecido objetivos muy específicos.

Las tres etapas que pueden definir el sistema educativo del país, de manera cronológica, son las siguientes: el periodo de la Reforma, el periodo de 1958 a 1970 y el inicio de la educación a distancia.

La presente investigación presenta una investigación cronológica con las aportaciones más considerables de cada una de estas etapas, así como un análisis de la realidad y los cambios que ha traído consigo la implementación de la virtualización en el sistema educativo, paradigma también conocido como "educación a distancia".

\section{El sistema educativo en México: una mirada a la historia}

Si tuviéramos que establecer una definición para "sistema educativo", podríamos decir que es una estructura que busca la enseñanza a través de ciertas reglas, relaciones, estructuras y medidas diseñadas por el Estado de un país y que es fomentada a través de instituciones y organismos, que ejercen, regulan, financían o prestan servicios para ello.

Debido a que este sistema surge a partir de las políticas públicas generadas por el Estado de un país, los cambios políticos afectan directamente a la educación de este. México ha atravesado por diversos periodos de cambios, algunos más significativos que otros.

\section{A. El sistema educativo en México durante el periodo de Reforma, 1857 a 1861.}

Benito Juárez, que gobernaba en aquel momento, implementó las leyes de reforma que transformaron significativamente la educación del país.
Dentro de ellas, afectó a los artículos $3^{\circ}$., $5^{\circ}$., $7^{\circ}$., $13^{\circ}$., $27^{\circ}$. y $123^{\circ}$. Relativos a la enseñanza libre, la privación del voto al clero, la supresión del clero al monopolio de la educación y la separación de la iglesia y el estado [2].

\section{B. El sistema educativo en México durante el periodo de 1958 a 1970.}

De acuerdo con (Muñoz Izquierdo, 1973), México atravesó por una serie de cambios muy relevantes en el periodo de 1958 a 1970 , esto durante los periodos de gobierno de los presidentes Adolfo López Mateos y Gustavo Díaz Ordaz [1].

Algunos de los más fundamentales son presentados en la Tabla 1.

\section{El surgimiento de la virtualización y los ambientes virtuales.}

De acuerdo con el portal de Azure de Microsoft, la virtualización se puede definir como "una herramienta para la creación un entorno informático simulado, o virtual, en lugar de un entorno físico [3]".

La virtualización se divide en cuatro categorías específicas: virtualización de escritorio (permite que un servidor centralizado ofrezca y administre escritorios indivualizados), virtualización de recursos de red, virtualización de software, y, finalmente, la virtualización del almacenamiento digital [3].

Si bien la tecnología de virtualización surgió en los años sesenta, fue hasta el año 2000 que comenzó a ser realmente adoptado. Durante los años 90, la mayoría de las empresas contaban con servidores físicos y pilas de Tecnologías de la Información de un mismo proveedor, que no permitían ser ejecutadas en otros tipos de hardware. Cuando la necesidad de mayor espacio o de mejor procesamiento, pero cuya búsqueda implicaba pocos recursos económicos para tener el hardware propio, es cuando comienza a popularizarse la virtualización [4]. 


\section{Cambio educativo}

\begin{tabular}{|lr|l|}
\hline $\begin{array}{l}\text { Demanda } \\
\text { potencial } \\
\text { escolaridad. }\end{array}$ & por & $\begin{array}{l}\text { La demanda educativa, } \\
\text { principalmente primaria y } \\
\text { secundaria, se incrementó } \\
56.4 \%\end{array}$ \\
\hline $\begin{array}{l}\text { Financiamiento del } \\
\text { sistema escolar. }\end{array}$ & $\begin{array}{l}\text { El financiamiento en la } \\
\text { educación pasó del 1.8\% al } \\
2.7 \% \text { del Producto Interno } \\
\text { Bruto (PIB) nacional. }\end{array}$ \\
\hline $\begin{array}{l}\text { Distribución de } \\
\text { oportunidades } \\
\text { escolares y sociales }\end{array}$ & $\begin{array}{l}\text { La posición social, el grado } \\
\text { de urbanización, y las } \\
\text { características geográficas } \\
\text { comenzaron a no ser } \\
\text { impedimento para estudiar, } \\
\text { aunque prevalecían las } \\
\text { preferencias a los sectores } \\
\text { privilegiados. }\end{array}$ \\
\hline
\end{tabular}

Tabla 1 Cambios fundamentales en el sistema educativo mexicano en el periodo de 1958 a 1970

\section{Educación media superior}

Hoy en día la educación media superior está prácticamente al acceso de cualquier persona y es que no se necesita acudir todos los días al plantel educativo donde los estudiantes acuden a tomar sus clases existen programas con cursos sabatinos para personas que no les es fácil asistir todos los días al plantel, sin embargo hay un programa mucho mejor que hoy en día está tomando gran importancia en no solo jóvenes si no también en adultos "Prepa en línea" es el nombre que la SEP ha dado a su programa creado el 15 de octubre del 2014, esta opción es muy viable para personas que por alguna razón no hayan tenido la oportunidad de realizar sus estudios de media superior o que bien no tengan la solvencia económica para estar inscritos en una institución de educación media superior [7].

Esta opción es totalmente gratis y los planes de estudio se acoplan de acuerdo a lo que el estudiante desee, solo es cuestión de tener acceso a internet y un dispositivo para conectarse.
En un principio se inscribieron 38,000 aspirantes de todo el país, de los cuales se seleccionaron 20,000 para realizar un curso propedéutico que tuvo inicio el 8 de septiembre del 2014 y finalizo el 4 de octubre del mismo año, estos números no son nada comparados con los datos del 2019 mostrados por la SEP en la NUMERALIA del 2019, en primera instancia se tiene un aumento del $735.9 \%$ en la matricula del nivel medio superior teniendo 147,180 estudiantes inscritos tan solo el año pasado donde el $38.6 \%$ de los estudiantes son hombres $(565,701)$ mientras que el $61.4 \%$ son mujeres $(90,479)$ lo cual deja por entendido que este modelo de educación media superior es muy llamativo para las personas [8].

Las cifras son arrasadoras respecto a años pasados, hace años muchas personas no veían como una opción esta modalidad, sin embargo hoy en día es la principal opción para personas que no pudieron continuar con sus estudios de manera regular, actualmente la matrícula de Prepa en línea tiene una participación de jóvenes de 13 hasta 29 años de 74,757 (50.8\%) del total de estudiantes mientras que la participación de adultos de 30 años o más es de 74,423 (49.2\%) del total de estudiantes, en pocas palabras esta opción no solo es llamativa para jóvenes si no que los adultos también se están interesando por esta opción de estudios de nivel medio superior donde la cifra de interesados en el año pasado en las 18 convocatorias que la SEP lanzo durante el transcurso del año fue de 572,288 lo cual es una cifra increíble en comparación a otras modalidades para estudiar.

Esta modalidad tiene presencia en toda la República Mexicana y no exenta a ningún grupo social ya que incluso personas con discapacidades tienen presencia en esta modalidad teniendo en su matrícula a 4,029 (2.7\%) estudiantes con discapacidad visual, $1,036(0.7 \%)$ con discapacidad motriz y 634 $(0.4 \%)$ con discapacidad auditiva [7].

Por último, la cifra de egresados es de 14,404 estudiantes de los cuales el 35\% de ellos decidieron cursas estudios universitarios. 
Y todo esto se debe a la virtualización de la educación en el país, mediante la implementación de las TIC's en la educación estamos avanzando y creando nuevas oportunidades para que todos los habitantes en México puedan tener a su alcance la educación del nivel medio superior y que así en algunos años la mayoría de la población en el país pueda tener estudios de nivel medio superior sin la necesidad de acudir a un plantel todos los días a tomar sus clases.

Si bien en el país las TIC's en la educación no son tan utilizadas se está tratando de avanzar día a día para que a mediano o a largo plazo se brinden todas las facilidades para estudiar en línea, aún existen brechas por ejemplo con la cobertura de internet en distintas zonas e incluso faltan dispositivos para que personas se conecten a las redes y puedan realizar sus actividades en las distintas plataformas que existen. Se tienen que cubrir estas brechas que existen para así lograr completar el objetivo que se tiene.

\section{Educación superior}

Dentro de las nuevas tendencias de educación la Universidad Juárez Autónoma de Tabasco (UJAT) incorpora "la educación a distancia" como nuevo método de aprendizaje en la división Académica de ciencias Sociales y Humanidad en 2003, posteriormente en 2004 tres más adoptarían este método.

El modelo de aprendizaje se diseñó fundamentalmente en la concepción pedagógica constructiva y Humanista del aprendizaje contando con 5 características para los estudiantes a distancia [9]:

- El estudiante desarrolla su aprendizaje bajo la orientación del docente tutor.

- Se combinan estrategias, métodos y medios técnicos y didácticos.

- Se aprende con la orientación de guías didácticas posibilitando la autoevaluación.

Existe capacidad de aprender más allá de un aula, eliminando las barreras de tiempo y espacio por lo que no implica la asistencia a clases.
- Garantiza al estudiante la misma calidad de aprendizaje que en la modalidad escolarizada.

Esto se realizó para para centrarse en el estudiante que por motivos personales tenga que trabajar y a su vez desea cursar o concluir una carrea profesional sin tener que apartarse de sus actividades laborales, ofreciendo una opción alterna para su educación superior. La modalidad de este aprendizaje se caracteriza por la responsabilidad de enseñar, generar y aplicar los conocimientos; además haciendo uso de las tecnologías de información y comunicación mediando a profesor y estudiante en una conversación potenciando el aprendizaje.

En el año 2014 la UJAT inaugura centros de educación a distancia en los municipios de Jonuta y Jalapa para que la ecuación llegue a puntos importantes que se encuentran a una distancia lejana de Tabasco; contando a un total de 81 estudiantes registrados en este modelo de aprendizaje. [9]

En la actualidad la UJAT cuenta con 3 tipos de proceso de enseñanza: presencial, a distancia y semipresencial; estos diferencian en su contenido y orientación didáctica por motivo de que los estudiantes metan son diferentes. En el proceso de enseñanza de los estudiantes a distancia se observa que estos poseen un mayor sentido de responsabilidad, innovación, son autodidactas, auto sugestivos y contando con un mayor compromiso al momento de repasar el material didáctico.

\section{La implementación de la virtualización en la educación: retos para los sistemas virtuales}

La educación virtual, que también es conocida como E-learning (EV) es una de las formas de enseñanza a distancia con uso predominante del Internet como medio tecnológico; La EV es un resultado de procesos convergentes en el campo de las TIC y en la evolución de las organizaciones educativas en las cuales aparecían las necesidades de reforzar los efectos educativos y simultáneamente, de crear nuevos dispositivos de teleeducación o educación a distancia. 
Las instituciones educativas lograron en un lapso relativamente breve de tiempo durante la segunda mitad de los años noventa, que el Elearning ocupara un lugar prestigioso en el espacio de la educación; Haciendo que cada vez más las universidades fueran incorporando esta tecnología en su quehacer junto con empresas de alcance global; que advirtieron que la nueva tecnología resultaba ventajosa para las funciones de formación y aprendizaje fenómeno de educación que requería su personal.

Cada día que pasa aumenta el desarrollo y aparición de nuevas tecnologías para la información y comunicación, además de que se posibilita más su acceso por su baja de costos y facilidades de uso; que de tal manera hemos visto el surgimiento de la realidad aumentada, internet de los objetos, la robótica, los vehículos autónomos y otros más que surgen con el pasar del tiempo, virtualizar supone hoy, la gestión del entramado entre los entornos de aprendizaje físicos y digitales, considerando que en unos y en otros hay mediación de las TIC para la representación o evocación de los objetos de conocimiento y el modelamiento de las interacciones para el aprendizaje.

La educación virtual lleva consigo diferentes retos, algunos enunciados a continuación:

\section{A. Reto 1: La diferencia entre la forma y el fondo.}

La diferencia entre forma y fondo, tan valiosa para nuestro pensamiento habitual ha sufrido confusiones desde que asumimos que el medio es el mensaje, lo que ahora podemos traducir como que la tecnología en ella misma.

\section{B. Reto 2: Ya no al diseño pedagógico sino epistemológico.}

Para continuar el acercamiento al tema Manuel Cebrian de la Sema afirma que "el poder en el aula ya no está en el control de la información, sino, más bien, en la capacidad de construir el conocimiento o en la capacidad de permitir crear un verdadero autoaprendizaje tutorizado en nuestros estudiantes: la información ya no es conocimiento'.

\section{Reto 3: La tecnología como contenido del pensamiento.}

Los estudiantes de sistemas de educación virtual deben poseer conocimientos y habilidades preliminares en el orden del manejo de la tecnología de comunicación y procesamiento de información.

\section{Reto 4: el tránsito de la descripción informativa a la hermenéutica de los datos y de los hechos.}

Los hechos, los datos de la historia, los textos y los comportamientos cotidianos son en ellos mismos, silentes, dado que no vienen con manuales explicativos ni con aproximaciones a su significado o sentido.

\section{E. Reto 5: el aprendizaje colaborativo y dialógico en cursos masivos.}

El trabajo colaborativo constituye una de las premisas de los cursos en línea, lo que es, de inicio, paradójico, en la medida que cada uno de los estudiantes efectúa su propia gestión formativa desde el aislamiento de su oficina o de su estudio; la mayoría de los estudiantes prefiere los cursos presenciales justamente por la realimentación directa, inmediata y en tiempo real; por el contrario, en los cursos en linea la realimentación entre estudiantes y la retroalimentación que proviene del profesor, se efectúan en tiempos diversos, si bien dentro de márgenes muy delimitados.

\section{F. Reto 6: el aislamiento del aprendizaje.}

Es necesario recordar que destaca un inevitable sentimiento de aislamiento y soledad que se manifiesta en la urgencia de encontrar la inmediata respuesta del profesor o del tutor ante sus dudas, preguntas y aportaciones, como si el profesor mantuviera su presencia continua en el otro lado de la línea.

\section{G. Reto 7: ruptura y conversión cognoscitiva.}

Asumimos que todo aprendizaje autogestivo es indudablemente, una conversión precedida de una ruptura cognoscitiva. No nos referimos a las rupturas epistemológicas de la historia en las que los paradigmas imperantes dejaron de ofrecer explicaciones a los temas y problemas humanos del momento, y por ello generaron cosmovisiones radicalmente diferentes. 
H. Reto 8: Las competencias sociales y humanas.

El modelo de cursos en línea asume como parte importante el trabajo colaborativo, como ya se ha dicho, pero asume también la formación de competencias de carácter social y humano que más difícilmente pueden alcanzarse. La dificultad se explica porque dichas competencias deben partir de una sensibilización cognoscitiva ante los problemas sociales, pero deben aterrizarse en gestiones específicas, en las que se noten las evidencias de su logro. Ante esta circunstancia importan las preguntas por la posibilidad de la humanización y formación ciudadana y la del desarrollo de habilidades para la realización profesional y la felicidad cotidiana.

\section{Reto 9: Educación virtual y TIC para la dignidad y la vida humanas.}

Seguiremos viviendo por mucho tiempo en el contexto de la educación virtual, el desarrollo de competencias y la sociedad del conocimiento, pero por ello mismo el siguiente reto que nuestra civilización propone es el referente humano de toda TIC y de todo aprendizaje virtual. De suyo la preocupación de todo modelo educativo es el hombre y la colectividad y en ello muchos coincidimos, como el filósofo alemán Keiner.

\section{J. Reto 10: El mito de la ligereza de los cursos y los riesgos de deshonestidad académica.}

Dada la distancia real entre el profesor y los estudiantes, lo mismo que la distancia entre estudiantes, ubicados en diversas ciudades y países, no hay manera de identificar personalmente el aprovechamiento específico de cada uno. Tampoco existe una manera de identificar que el alumno efectúe sus trabajos y no un amigo, como solía ocurrir en la secundaria. No es difícil reconocer trabajos que no son sino documentos bajados de alguna página de Internet e incluso existen mecanismos para saberlo.

\section{K. Reto 11: La carga simbólica de los cursos en línea y de la educación.}

A juzgar por su dimensión tecnológica, los cursos en línea poseen una alta carga simbólica, si bien, dentro de los márgenes del formalismo lógico, matemático e informático.
Mediante iconos, password y códigos comprendemos y accedemos a las computadoras, a los servidores de Internet, a los programas y a todos los comandos. El buen estudiante de cursos en línea debe ser un experto en la semiótica de las máquinas.

\section{¿La educación a distancia es para todos?}

Sobre el cuestionamiento acerca de quienes tienen acceso a la educación a distancia, consideramos que habría que pensar en las tareas pendientes, en las promesas -no cumplidas aún que se hacen en nombre de la educación a distancia, entre ellas:

- La atención a los históricamente marginados por sus precarias condiciones de existencia que, si no tienen siquiera acceso a los mínimos recursos vitales de subsistencia, menos a la educación institucionalizada.

- Desarrollar y llevar a cabo estrategias especiales para personas ciegas, sordomudas, con discapacidades motrices y otras, cuyas debilidades orgánicas les impiden acceder con las mediaciones convencionales.

- Dotar a los programas académicos de la flexibilidad y adaptabilidad que les permitan adecuarse a las condiciones de vida de quienes aspiran a su estudio.

\section{Conclusiones}

Desde luego, aunque la cultura de la innovación académica ha avanzado por la vía de la educación a distancia y las nuevas tecnologías digitales, falta mucho por hacer en el ámbito de las políticas educativas, la gestión organizacional y las prácticas académicas y administrativas cotidianas. En esa misma dirección, observar e investigar más, desde una visión integral, los factores económicos, políticos y culturales que repercuten en el desarrollo de la educación a distancia, su posicionamiento social e institucional y trascendencia, como base para tomar las decisiones pertinentes y apropiadas.

GALLEGOS-GALINDO, Heriberto, AGUILERA-GUTIÉRREZ, Enrique, DOMÍNGUEZ-PIZANO, Luis y CASTRO-VALENCIA, Alan. Virtualización de la educación media superior y superior en México: cambios y realidades. Revista de Educación Técnica. 2020 
Además de cumplir lo incumplido, la educación a distancia tiene la oportunidad de incidir en otras modalidades educativas; más allá de las modalidades, ser un ámbito de experimentación educativa. Para nuevos proyectos con visión de futuro, veo el presente como el pasado de ese futuro deseado.

Somos conscientes de que el porvenir es impredecible, por lo tanto, de poco o nada sirve decidir cuáles modelos de estructuras organizacionales serán los idóneos, cuáles conocimientos específicos serán necesarios para incluirlos en los currículos escolares, o cuáles destrezas laborales concretas serán útiles para las nuevas profesiones. De ahí que una educación para el futuro requerirá una preparación para lo incierto y cambiante, basada en la creatividad, gestión significativa del conocimiento, además de actitudes y aptitudes que hagan posible la convivencialidad.

\section{Referencias}

[1] Muñoz Izquierdo, Carlos. Evaluación del Desarrollo Educativo en México (19581970) y Factores que lo han Determinado. Revista del Centro de Estudios Educativos (México), vol. III, núm. 3, 1973, pp. 11-46.

[2] Torres Hernández, Adolfo. Juárez y la libertad de enseñanza. Milenio digital. Consultado el 09 de febrero de 2020. url: https://www.milenio.com/opinion/alfonsotorres-hernandez/apuntes-

pedagogicos/juarez-y-la-libertad-deensenanza.

[3] Microsoft Azure. ¿Qué es la virtualización? Consultado el 09 de febrero de 2020. url: https://azure.microsoft.com/es$\mathrm{mx} /$ overview/what-is-virtualization/

[4] RedHat. ¿Qué es la virtualización? Consultado el 09 de febrero de 2020. url: https://www.redhat.com/es/topics/virtualiza tion/what-is-virtualization

[5] Coronado Ramírez, G. C. R. Gerardo. (2017). Educación a distancia en México (Ed. rev.). Consultado el 09 de febrero de 2020. url: http://bit.ly/39gA3Ew
[6] Micheli Thirión, J. M. T. Jordy, y Garrido Noguera, C. G. N. Celso. (2005). La educación virtual en México: universidades y aprendizaje tecnológico (Ed. rev.). Consultado el 09 de febrero de 2020. url: http://bit.ly/2tBxnlz

[7] Cudi, CD. (2014). Presentación oficial de la Prepa en Línea SEP (Ed. Rev.). Consultado el 25 de febrero de 2020. url: https://www.cudi.edu.mx/noticia/presentaci on-oficial-de-la-prepa-en-linea-sep

[8] Secretaria de Educación Publica, SEP. (2020). Numeralia 2019 (Ed. Rev.). Consultado el 25 de febrero de 2020. url: http://www.prepaenlinea.sep.gob.mx/image s/assets/Numeralia2019.pdf

[9] Autónoma de Tabasco, Universidad Juárez. (2014). Interior adentro (Ed. rev). Consultado el 16 de febrero de 2020. url: http://www.test.ujat.mx/interioradentro.asp $\mathrm{x} ? \mathrm{ID}=15379 \& \mathrm{NODO}=229$ 